\title{
EFFET DE L'ACTIVITÉ ENDOCRINE CÉRÉBRALE SUR LA MATURATION DE L'OVOCYTE DES NÉREIDIENS (ANNÉLIDES POLYCHÈTES). ASPECTS CYTOLOGIQUES ET BIOCHIMIQUES
}

\author{
M. PORCHET et A. DHAINAUT \\ Laboratoire d'Endocrinologie comparée des Invertébrés associé au C. N.R. S., no 148, \\ Laboratoire de Biologie animale, \\ Université des Sciences et Techniques de Lille, \\ B. P. 36, 59650 Villeneuve d'Ascq (France)
}

\section{RÉSUMÉ}

La maturation ovocytaire des Néréidiens a été abordée sous l'angle cytologique, biochimique et endocrinologique. Dans une première phase, apparaissent les réserves lipidiques et le vitellus protéique. Le nucléole présente alors un aspect granulaire. Pendant le stade suivant, les dictyosomes élaborent des substances polysaccharidiques riches en acides uroniques et en sulfate. L'analyse biochimique de ces ovocytes montre que leur teneur en glucides est doublée par rapport au stade précédent. Le nucléole perd sa structure granulaire et devient fibrillogranulaire.

La séquence complète de ces synthèses n'est réalisée que sous un conditionnement hormonal précis. Elle exige une décroissance régulière de la production endocrine du cerveau qui s'annule lors de la submaturité. L'hormone cérébrale est inhibitrice de la maturation sexuelle mais sa présence, à des niveaux d'activité bien définis, est nécessaire au bon développement du métabolisme ovocytaire.

Parmi les Annélides Polychètes, c'est chez les Néréidiens que la sexualité est le mieux connue. Ces vers marins, qui ne se reproduisent qu'une fois, meurent dans les jours qui suivent 1'essaimage au cours duquel se fait 1'émission des produits génitaux. Selon les genres, la durée de vie est de I, 2 ou 3 ans (HERPIN, I925).

Trois états successifs caractérisent la sexualité de ces animaux :

I) absence de produits génitaux (état dit, par simplification, " asexué ") ; 
2) gamétogenèse ;

3) maturité génitale et reproduction de l'animal.

Au moment de la reproduction, la plupart des espèces de Nereis subissent d'importantes modifications morphologiques et anatomiques. On assiste à la transformation des parapodes et corrélativement à des remaniements musculaires dans les segments correspondants. A la fin de cette phase appelée épitoquie, la Nereis est devenue une Heteronereis capable de nager en pleine mer où s'effectue l'essaimage.

Depuis les travaux de Durchon (I948 à I952), de nombreuses recherches ont été entreprises sur ce sujet (HAUENSCHILD, I965; ClARK, I966; DURCHON, I967a). De l'état actuel de ces recherches, il est possible de dégager les faits suivants :

ro Un centre neuroendocrine situé dans les ganglions cérébroïdes sécrète une hormone qui inhibe la gamétogenèse et les transformations hétéronéréidiennes (Durchon, I956 à I960 ; CLARK, I956 ; HAUENSCHIL, I963-I965 ; CHOQUET, I962 ; Clark et Ruston, I963 $a$; BoIl, Y-MARER, I962 ; DhaInaUt, I964 à I970 ; DhaINAUt et Porchet, I967 ; Porchet, I967-I970 ; MALECha, I967 ; Schroeder, I97I).

$2^{\circ}$ L'activité endocrine qui régit la sexualité est d'origine uniquement prostomiale (DurchoN, I952; HAUENSCHILd, I956 ; GoldING, I967 ; CrARK, r969) et il n'existe probablement qu'une seule hormone (PORCHEr, I972).

$3^{\circ}$ Cette activité endocrine décroît régulièrement au cours du cycle vital. Importante pendant la phase " asexuée ", elle devient nulle au moment de la reproduction (DURCHON et PorcheT, I97I). Le ralentissement de la production hormonale est influencé par la photopériode (HAUENSchild, I955, I960 $b$ ), la température (DuRCHON et PORCHET, I97I) et réglé par une rétroaction génitale (DURChON, I952; Porcheit, I967 ; Porchet et Durchon, I968; Porchet et Cardon, I972).

L'apparente simplicité du cycle de reproduction, la présence d'une seule hormone à site de production unique font des Néréidiens un matériel de choix pour l'étude des problèmes relatifs à la maturation génitale.

\section{I. - LES DIFFÉRENTS STADES DE L'OVOGENÈSE DES NÉRÉIDIENS}

Il n'existe pas de gonade ni de cellules folliculaires chez les Néréidiens. Les gamètes sont libres dans le liquide cœlomique. Il est donc aisé de prélever les ovocytes sur le vivant. L'état génital d'un individu est déterminé par l'étude biométrique du diamètre ovocytaire moyen.

Il est possible de distinguer deux phases successives dans le métabolisme ovocytaire (fig. I) :

- de 30 à I20 $\mu$ de diamètre, l'ovocyte élabore des substances de réserves d'abord lipidiques puis protéiques ("vitellus protéique " des auteurs);

- de I30 $\mu$ à la maturité (diamètre ovocytaire de $250 \mu$ ), 1'ovocyte synthétise des réserves essentiellement glucidiques. Une étude cytochimique montre que cellesci présentent initialement une réaction métachromatique aux colorants basophiles 


\section{activité endocrine}

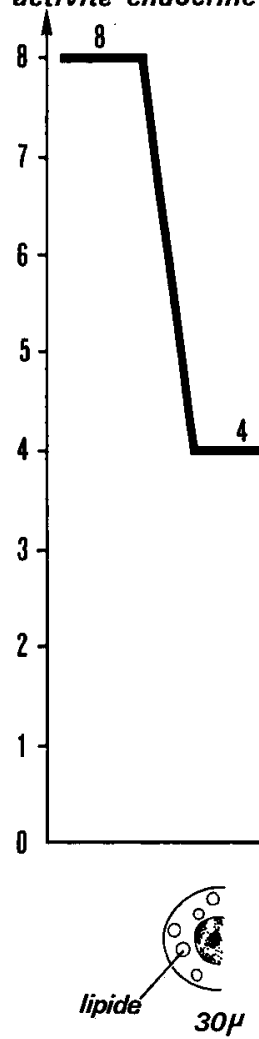

$30 \mu$
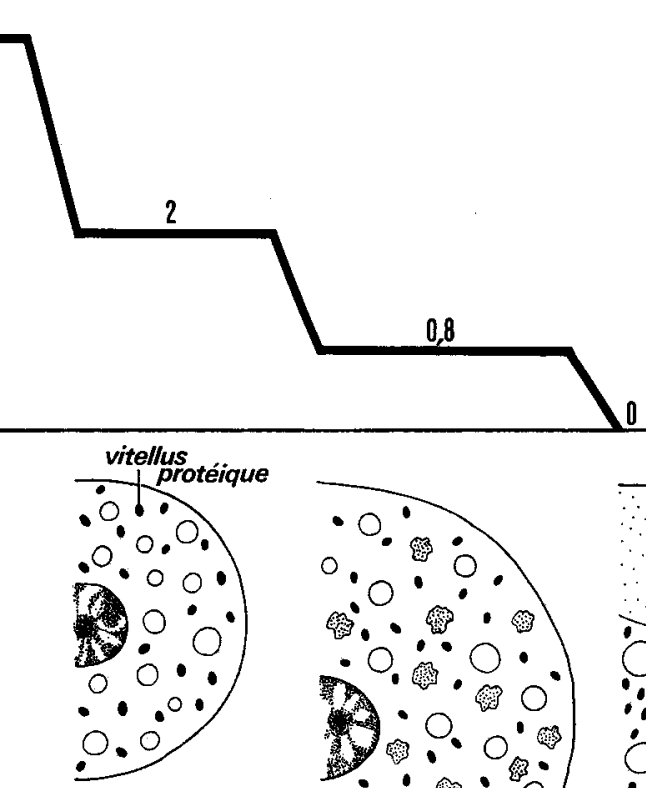

60 à $130 \mu$
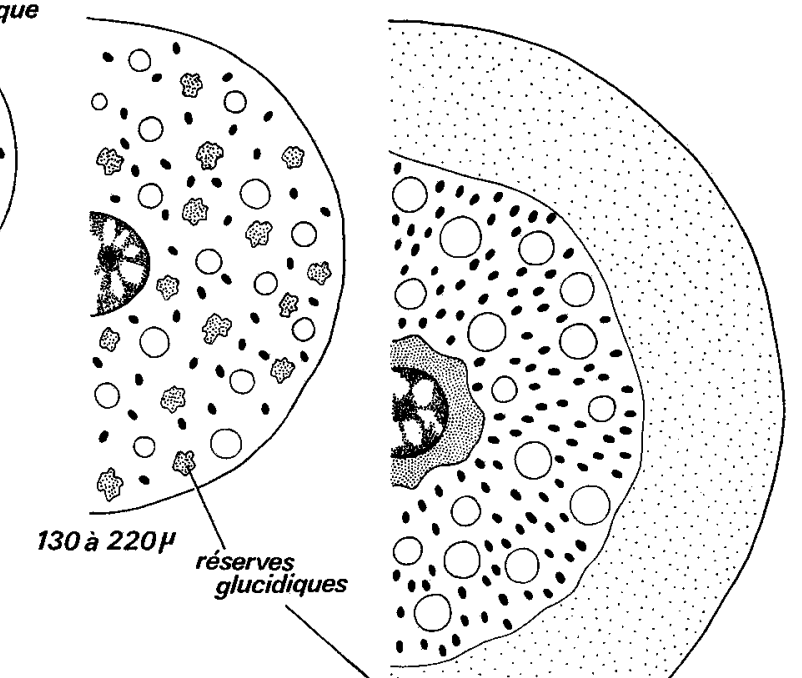

maturité $250 \mu$

FIG. I. - Correspondance entre les valeurs de l'inhibition cérébrale, après dosage biologique, et le métabolisme ovocytaire chez $\mathrm{P}$. cultrifera 
(bleu de toluidine par exemple) puis (I80-250 $\mu$ ) réagissent positivement aux réactions qui semblent indiquer la présence des mucopolysaccharides acides (bleu alcian, muci carmin...) (Porchet et Dhainaut, I969).

Au moment de la maturité génitale, l'ovocyte subit de profonds remaniements. Les polysaccharides se regroupent à la périphérie du cytoplasme où ils constituent une gangue épaisse. Celle-ci sera totalement expulsée lors de la fécondation. L'ARN cytoplasmique se concentre en une auréole périnucléaire tandis que globules vitellins et lipides occupent la partie moyenne de la cellule.

Ces synthèses cytoplasmiques sont placées sous la dépendance de l'activité inhibitrice cérébrale.

\section{II. - LE CONDITIONNEMENT ENDOCRINE DE L'OVOGENÈSE}

\section{A. - Nature de l'hormone cérébrale}

Les ganglions cérébroïdes sécrètent une hormone qui contrôle le développement ovocytaire. Cette hormone exerce une double action : d'une part, elle est inhibitrice de la croissance des ovocytes ; d'autre part, elle est indispensable à leur métabolisme (PORCHET, I970).

\section{L'hormone est inhibitrice.}

C'est la propriété fondamentale de l'activité endocrine cérébrale : la décérébration de l'animal provoque l'accélération de la croissance ovocytaire. Dans quelques cas (à partir d'un certain degré de maturité génitale), il est même possible d'obtenir de cette façon une maturation sexuelle précoce. Les ovocytes ne sont pas les seules cellules à réagir à la levée de l'inhibition : en fait, tout le soma est sensible au conditionnement anhormonal. Les transformations hétéronéréidiennes se produisent alors, le ver devient incapable de régénérer postérieurement (CASANOVA, I955 ; DURCHON, I956 $b$, I967 $b$; HAUENSCHIL,D, I96o $a$; Cl,ARK et al., I96o à I963 ; Gol,Ding, I967), et on assiste à une induction de la reproduction des sporozoaires parasites intracoelomiques (Durchon et VIVIER, I964; PORChET-HÉnNERE, I969).

Chez les Néréidiens, la maturation naturelle ou expérimentale est corrélative des transformations somatiques de l'épitoquie. D'après les résultats des expériences entreprises (HAUENSCHILD, I966 et I970 ; BOILLY-MARER, I962) il semble bien qu'une seule et même hormone cérébrale contrôle l'ensemble des phénomènes sexuels.

\section{L'hormone est nécessaire aux synthèses ovocytaires.}

Cette propriété de 1'hormone a été mise en évidence récemment. HAUENSCHILD (rg64) le premier, a montré qu'une Platynereis évoluant expérimentalement avec une très faible quantité d'hormone, subissait une maturation génitale et somatique plus rapide que les animaux évoluant sans cerveau, c'est-à-dire en condition anhormonale. Nous avons précisé ce mécanisme d'action chez Perinereis cultrifera (PoRCHET, r969). Dans de jeunes femelles, privées de leurs ganglions cérébroïdes, nous avons implanté des cerveaux à activité endocrine réduite, prélevés chez les individus 
âgés. Dans la moitié des cas, les ovocytes des individus opérés deviennent matures. Par contre, chez tous les témoins décérébrés (aucune greffe) les ovocytes après avoir augmenté de taille finissent toujours par dégénérer. L'absence totale d'hormone cérébrale conduit les jeunes ovocytes à la dégénérescence.

Cette dégénérescence affecte essentiellement les jeunes ovocytes (diamètre inférieur à I3o $\mu$ ) qui, dans les conditions naturelles, évoluent en présence d'une forte activité endocrine cérébrale. Si celle-ci vient à être supprimée (décérébration de l'animal), dans un premier temps la taille des ovocytes double mais le métabolisme cellulaire ne suit pas cette accélération : en particulier il n'y a pas de synthèse des polysaccharides du cytoplasme. Cette croissance n'est en fait qu'une hypertrophie cellulaire. Après 40 à 50 jours presque tous les ovocytes dégénèrent.

La suppression du facteur hormonal est moins dramatique pour les ovocytes plus âgés (diamètre supérieur à $I 30 \mu$ ) et qui ont amorcé leurs synthèses glucidiques. Dans ce cas, il leur devient possible d'atteindre la maturité.

\section{B. - Variation de l'activité endocrine cérébrale en fonction des stades de l'ovogenèse}

DuRchoN et PORChET (I970, I97I) ont récemment mis au point une technique de dosage biologique de l'activité endocrine cérébrale en fonction de l'état génital des Néréidiens ( ${ }^{1}$. Une centaine de ganglions cérébroïdes provenant d'une même catégorie d'animaux, sont broyés dans de l'eau bidistillée puis centrifugés. Le liquide surnageant, renfermant une certaine quantité d'hormone inhibitrice, est introduit dans un milieu de culture sur lequel sont placés des parapodes isolés de Nereis diversicolor mâles. Ces derniers servent de témoins et de test. En effet :

- privés d'hormone cérébrale, les spermatocytes qu'ils contiennent se transforment en spermatozoïdes en 6 jours de culture, en moyenne ;

- associés à une source permanente d'hormone inhibitrice (un ganglion cérébroïde) leurs spermatocytes n'évoluent pas pendant toute la durée de la culture (30 jours) ;

- lorsqu'on ajoute une certaine quantité d'hormone inhibitrice, issue du broyage des cerveaux, on constate un retard de la maturation des spermatocytes. Ce retard est proportionnel à la quantité d'hormone produite par chaque type de cerveaux que nous introduisons avec le liquide surnageant des broyats. En effet, un même volume de liquide surnageant induira des retards variables dans la maturation spermatocytaire suivant que les cerveaux broyés sont plus ou moins inhibiteurs in situ.

De ces tests nous pouvons tirer les enseignements suivants :

Io L'hormone produite par des cerveaux prélevés sur des femelles est la même que celle qui inhibe la spermatogenèse (HAUENSCHILD, I970; BOILLY-MARER, I962). Ainsi, des ovocytes implantés dans la cavité cœlomique de receveurs mâles évoluent normalement pendant plusieurs mois (PORCHET, I972). Des études statistiques ont

(1) La description technique complète de cette méthode de dosage est longue. Nous renvoyons aux publications antérieures: Durchon et Porchet (1970, 1971) et Porchet (x972). 
montré que, dans ces conditions, les ovocytes présenteront une croissance accélérée par rapport aux ovocytes non transplantés. Il est ainsi possible d'obtenir un véritable " hermaphrodisme " expérimental. Il n'existe donc pas de profondes différences physiologiques entre les deux sexes chez les Néréidiens.

$2^{\circ}$ Mais si l'hormone cérébrale est la même chez les mâles et chez les femelles il existe une différence d'ordre quantitatif. Les ganglions cérébroïdes des individus femelles produisent environ quatre fois plus d'hormone inhibitrice que ceux des mâles.

$3^{\circ}$ L'activité endocrine cérébrale décroît régulièrement durant l'ovogenèse (fig. 2). Cette décroissance, de type logarithmique, est fonction de la taille moyenne

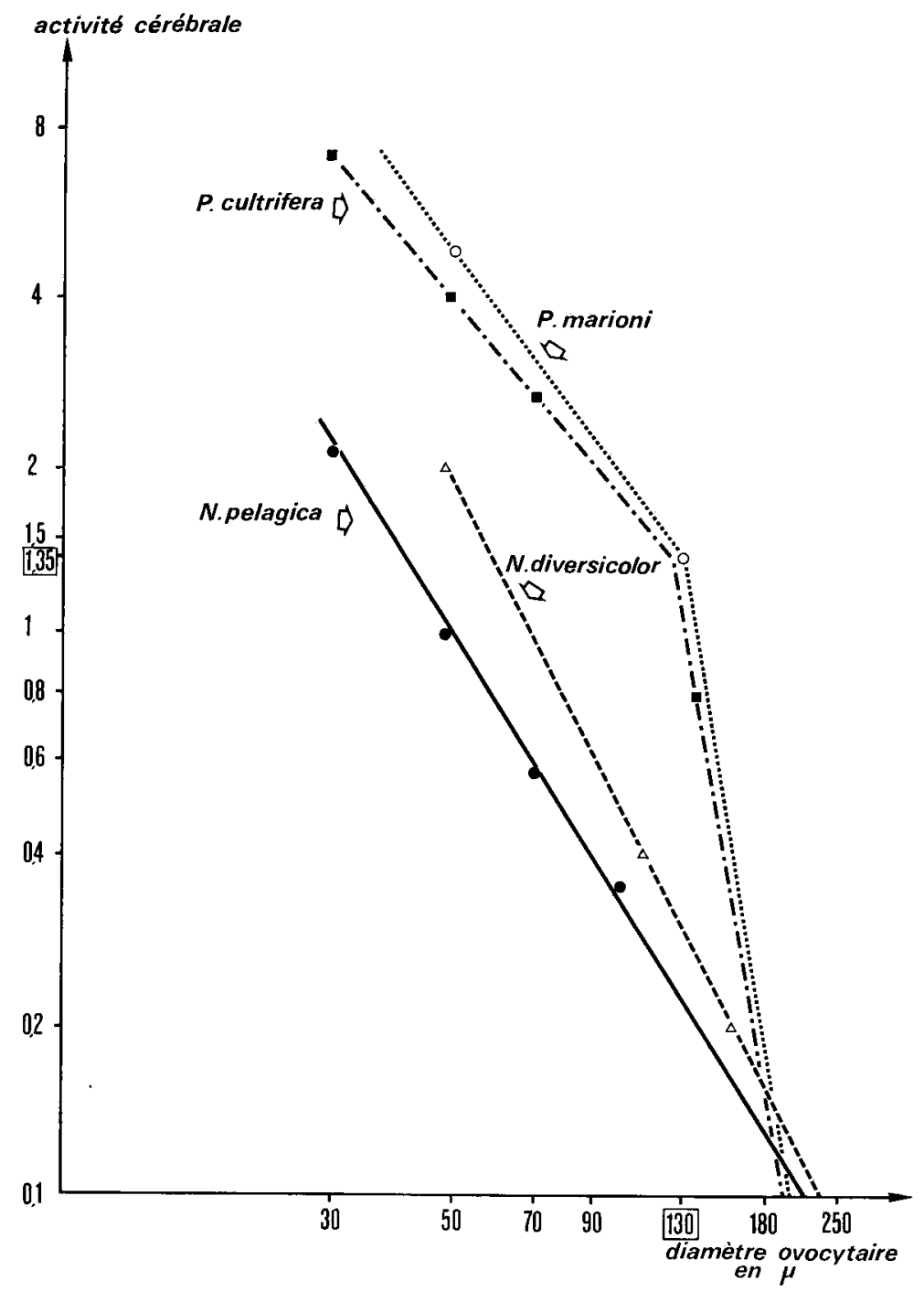

FIG. 2. - Représentation logarithmique de l'activité cérébrale en fonction de la taille ovocytaire chez quatre espèces de Néréidiens (d'après Porchet, I972). Explications dans le texte 
des ovocytes. Ainsi plus le diamètre ovocytaire augmente, plus le taux hormonal s'abaisse. Celui-ci finit par s'annuler au moment de la submaturité (taille ovocytaire supérieure à $\mathrm{I} 80 \mu$ ).

Ces données quantitatives permettent de définir deux types d'ovogenèse :

- celles des Nereis, chez lesquels la décroissance hormonale est constante et régulière et s'effectue sur deux années;

- celles des Perinereis où le ralentissement de l'activité endocrine se fait en deux temps. Jusqu'à une taille ovocytaire de I30 $\mu$ la baisse de l'inhibition est faible ; au-delà de ce stade la décroissance est brutale. Ces animaux vivent trois ans et l'activité hormonale inhibitrice de leurs cerveaux est plus grande que celle des Nereis. La durée totale de l'ovogenèse (de quelques mois à deux années selon les espèces) est fonction de la quantité d'hormone présente dans les cerveaux des différents Néréidiens.

\section{III. - ASPECTS CYTOLOGIQUES DE L'OVOGENÈSE DES NÉRÉIDIENS}

A chaque état endocrine correspond un état ovocytaire. Ainsi l'ovogenèse de $P$. cultrifera s'effectue suivant le schéma général décrit précédemment. Afin de permettre l'édification de chaque inclusion cytoplasmique (lipides, vitellus protéique, polysaccharides), les cerveaux produisent une quantité d'hormone bien définie et mesurable par dosage biologique (fig. I). Lorsque le taux hormonal est élevé, le cytoplasme élabore les lipides ainsi que le vitellus protéique ; lorsque le taux hormonal est faible ou nul apparaissent les réserves glucidiques.

Il est possible en changeant le conditionnement endocrine des ovocytes de moduler certaines synthèses.

\section{A. - L'hormone cérébrale induit la formation des réserves cytoplasmiques ovocytaires}

L'apparition d'une substance de réserve cytoplasmique dans l'ovocyte des Néréidiens est conditionnée par une double fonction de l'hormone cérébrale :

- d'une part, l'activité endocrine du cerveau doit diminuer. Expérimentalement, il est possible de maintenir constant, ou même d'augmenter, le taux hormonal du milieu intérieur de l'animal. Il suffit de greffer plusieurs cerveaux d'individus plus jeunes que le receveur. Le métabolisme ovocytaire, la maturation génitale et même les transformations hétéronéréidiennes (DURCHON et CHOQUET, I96I) sont alors bloquées. Aucune inclusion cytoplasmique nouvelle ne peut apparaître. Dans de telles conditions il est possible d'augmenter la durée de vie de l'animal de quelques mois mais très généralement les ovocytes finissent alors par dégénérer ;

- d'autre part, il faut que l'activité hormonale du cerveau se stabilise à une valeur plus faible. Ce nouvel état endocrine induira une nouvelle synthèse au sein de l'ovocyte. Ainsi, il est possible de faire apparaître les réserves glucidiques dans les ovocytes de $60 \mu$ de diamètre, qui normalement en sont totalement incapables, en greffant dans la cavité cœlomique d'un jeune ver décérébré deux cerveaux provenant d'animaux dont les ovocytes sont en pleine synthèse des glucides de réserve 
( $50-\mathrm{r} 70 \mu$ ). L'ovocyte de $60 \mu$ double de taille et subit une maturation accélérée (fig. 3).

Les deux fonctions de l'hormone sont donc indispensables pour assurer un développement harmonieux de 1'ovocyte. Ainsi, la levée de l'inhibition cérébrale engendre presque toujours chez les jeunes ovocytes un ralentissement ou même l'arrêt des synthèses cytoplasmiques. BER'TOU'T et DHAINAUT (I97I) ont observé que l'élaboration du vitellus protéique de $N$. diversicolor est stoppée lorsqu'on décérèbre l'animal. Ces auteurs utilisent comme critère l'incorporation de trois acides aminés tritiés : la leucine, la lysine et l'arginine. Dans tous les cas, l'incorporation, intense chez les ovocytes témoins évoluant avec un taux hormonal élevé, cesse totalement I 6 jours après l'ablation des ganglions cérébroïdes.

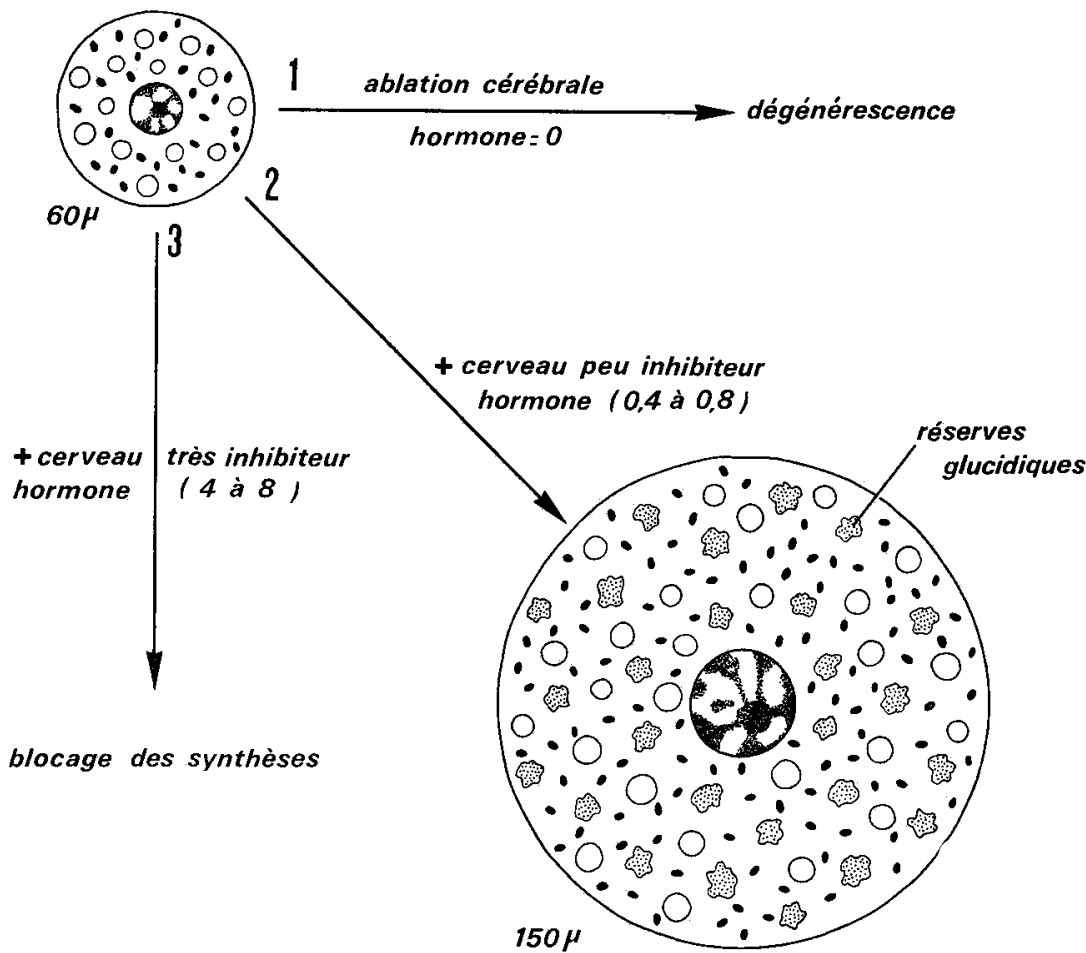

FIG. 3. - Infuence de divers conditionnements endocrines sur l'apparition des réserves glucidiques dans les ovocytes de P. cultrifera. Seule une activité hormonale réduite permet les synthèses polysaccharidiques

Les unités de l'activité hormonale sont celles de la figure 2

\section{B. - Évolution de l'appareil de Golgi et des inclusions cytoplasmiques}

I. Stade d'élaboration du vitellus protéique.

A ce stade, l'appareil de Golgi des ovocytes de Néréidiens est représenté par des dictyosomes d'une longueur d'environ $2 \mu$, situés à proximité de l'enveloppe ovocytaire et alignés parallèlement à cette dernière. Les dictyosomes ont une polarité 
bien nette. Les saccules de la face proximale montrent un aspect dilaté alors que ceux de la face distale sont réguliers et renferment un matériel dense.

Les globules vitellins en formation sont situés à proximité des dictyosomes. On y décèle de nombreuses vésicules morphologiquement identiques à celles élaborées par l'appareil de Golgi. Ces vésicules semblent matérialiser le processus par lequel s'effectue le transfert du matériel protéique depuis les dictyosomes jusqu'aux globules protéiques (fig. 4 et 5 ).

L'étude autoradiographique effectuée chez $N$. diversicolor (Dhainaut, I967) montre en effet qu'à la suite d'une incorporation de leucine- ${ }^{-3} \mathrm{H}$, le marquage est localisé initialement dans la zone ovocytaire corticale, au niveau des ribosomes. Trois à six heures après l'injection, les grains d'argent sont situés essentiellement au niveau du complexe golgien. Après 24 à 48 heures d'évolution, la radioactivité régresse fortement dans les dictyosomes; le marquage est limité à certains globules vitellins caractérisés par leur structure vésiculaire.

Ces résultats autoradiographiques montrent que les synthèses protéiques de la vitellogenèse 's'effectuent selon un schéma comparable à celui des cellules à sécrétion protéique exocrine (CARO et PALADE, I964; JAMIESON et PALADE, I967). Il soulignent le rôle de l'appareil de Golgi dans l'édification du vitellus.

\section{Stade d'élaboration des polysaccharides.}

Lors de l'apparition des polysaccharides (diamètre ovocytaire supérieur à I $30 \mu$ ), de profonds remaniements caractérisent les dictyosomes. Leur localisation reste identique mais leur dimension s'accroît considérablement et passe chez $N$. pelagica, d'une longueur d'environ $2 \mu$ (ovocytes jeunes) à 5 à $6 \mu$ en moyenne. Dans ces corps de Golgi, les saccules de la face distale sont dilatés. Ils renferment une substance peu dense aux électrons et ayant une structure fibrillaire. Les vacuoles provenant de la fragmentation des saccules distaux semblent établir, par leur morphologie, une filiation entre ces derniers et les plages polysaccharidiques du cytoplasme.

Cette évolution structurale est particulièrement nette chez $N$. pelagica et $P$. cultrifera. Chez $N$. diversicolor, l'élaboration des composés polysaccharidiques est faible. Pour cette espèce un processus évolutif similaire, mais plus complexe, conduit à la formation de deux types différents d'organites corticaux.

L'étude autorađiographique a été effectuée chez $N$. pelagica en utilisant du glucose- ${ }^{-3} \mathrm{H}$ comme précurseur (Dhainaut, I968). Pour des temps courts d'incorporation (expérimentation effectuée in vivo et in vitro), la radioactivité apparaît localisée uniquement au niveau de 1'appareil de Golgi. Son intensité est particulièrement élevée au niveau des saccules distaux. Pour des temps d'incorporation de plus longue durée (9 heures et 24 heures), le marquage apparaît successivement au niveau des vacuoles golgiennes puis dans les lobules polysaccharidiques. Ces résultats comparables à ceux de NEUTRA et LEBLOND (I966), semblent indiquer que la biosynthèse des polysaccharides complexes s'achève au niveau des saccules golgiens.

En conclusion, la croissance ovocytaire est caractérisée par l'évolution fonctionnelle de l'appareil de Golgi. Dans les jeunes ovocytes, les dictyosomes condensent les protéines élaborées au niveau des ribosomes et du réticulum endoplasmique et 


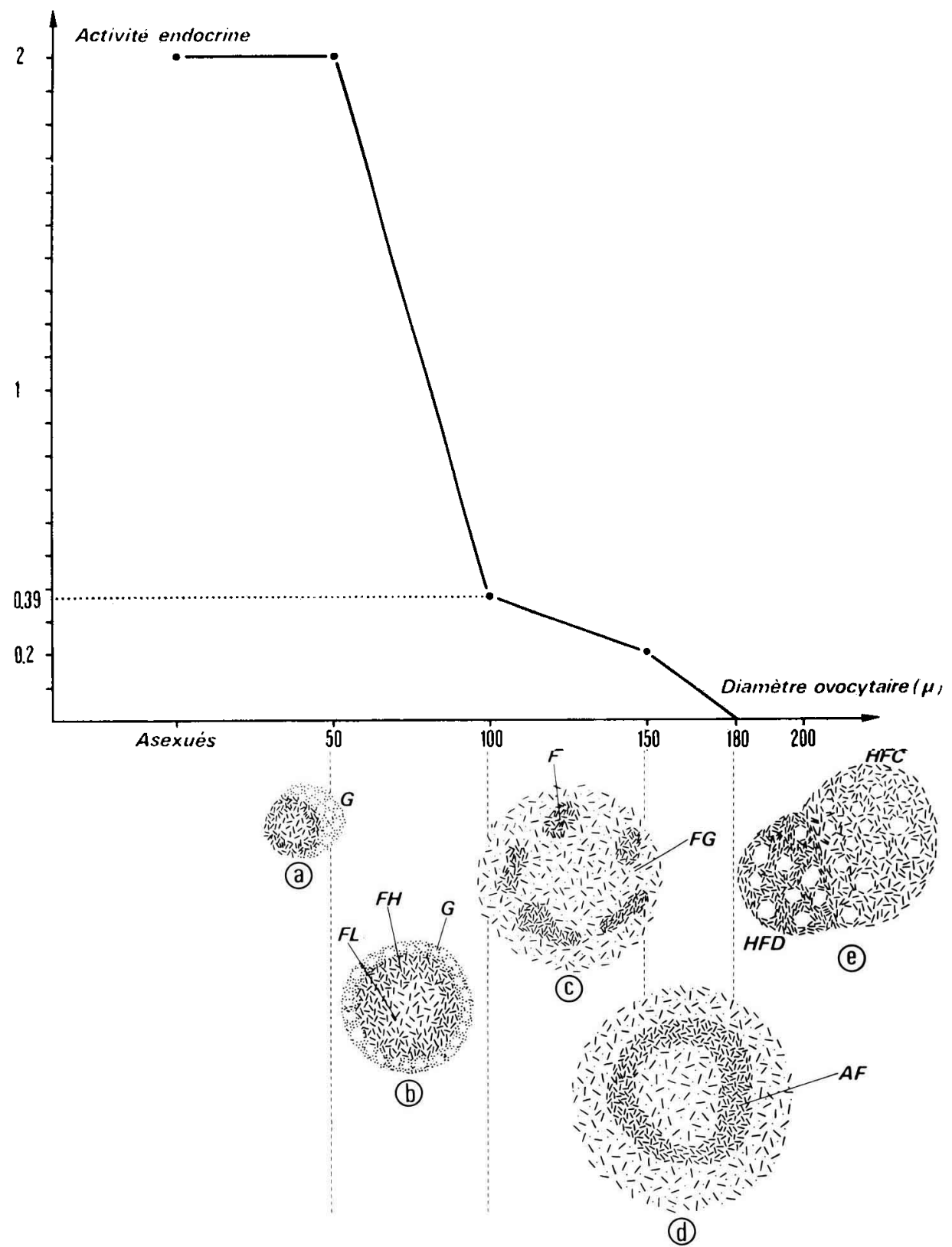

FIG. 6. - Relation, au cours de la croissance ovocytaire, entre la structure du nucléole et l'activité endocrine du cerveau.

(d'après BerTout, 1972)

$\mathrm{G}$ : composant granulaire ; $\mathrm{F}$ : composant fibrillaire ; $\mathrm{FL}$ : composant fibrillaire lacunaire ; $\mathrm{FH}$ : composant fibrillaire homogène ; $\mathrm{AF}$ : anneau fibrillaire ; $\mathrm{HFC}$ : hémisphère fibrillaire clair ; HFD : hémisphère fibrillaire dense. 
contribuent ainsi à l'édification du vitellus protéique. A l'approche de la maturité sexuelle, les dictyosomes sont l'objet de profonds remaniements. Ils interviennent alors dans l'élaboration de réserves riches en polysaccharides acides.

\section{C. - Évolution du nucléole}

Cette étude réalisée chez $N$. pelagica (DHarnaut, r972) et en collaboration avec BERTOUT chez $N$. diversicolor, montre qu'il existe une corrélation étroite entre la structure du nucléole et l'orientation des synthèses du cytoplasme. En schématisant les processus complexes de l'évolution nucléolaire on peut noter que le début de l'ovogenèse est caractérisé par la prédominance du composant granulaire et par l'existence de nombreuses extrusions de matériel nucléolaire vers le cytoplasme (Dhainaut, r964; Al, IEN, I967; TWEedel, I966). Ce transfert de substance a pu être suivi en autoradiographie à haute résolution par l'étude cinétique d'une incorporation d'uridine-- ${ }^{3} \mathrm{H}$ (DhaINaUT, I970). La fin des synthèses protéiques s'accompagne d'un remaniement de la structure du nucléole. Le composant granulaire se raréfie, le matériel fibrillaire présente une réorganisation importante. Une nouvelle configuration nucléolaire caractérisera la phase suivante des synthèses polysaccharidiques de l'ovocyte.

Les étapes de l'évolution nucléolaire coïncident étroitement avec les fluctuations de l'activité cérébrale. Cette étude réalisée chez $N$. diversicolor (BERTOUT, sous presse) est résumée dans le diagramme de la figure 6 . Cette évolution structurale du nucléole pourrait être liée au rôle régulateur joué par cet organite dans le transfert des ARN ribosomaux (Gould et SCHROEDER, I969) vers le cytoplasme. A titre d'hypothèse de travail, ces données suggèrent également que le nucléole pourrait constituer un site récepteur par l'intermédiaire duquel le contrôle hormonal s'exercerait au niveau de l'ovocyte.

\section{IV. - DONNÉES BIOCHIMIQUES}

Une étude biochimique a été entreprise sur les ovocytes isolés ainsi que sur le liquide cœlomique (SPIK et PORCHET, sous presse) de $P$. cultrifera. Nous nous sommes intéressés dans un premier temps à l'évolution des glucides ovocytaires (tabl. I).

La teneur en glucides est faible pendant la synthèse de vitellus protéique (I5, 8 p. IOo) ; elle est doublée $(28,4$ p. IOo) lorsque le cytoplasme ovocytaire élabore les réserves glucidiques et présente une nouvelle augmentation significative lors de l'épitoquie de l'animal (34,6 p. I00).

La cytochimie et la microscopie électronique ont révélé l'existence de trois constituants principaux renfermant des glucides :

- le vitellus protéique, de nature chimique très complexe;

- les plages polysaccharidiques (réactions colorimétriques positives au PAS, métachromasie, bleu alcian) ;

- le glycogène. 
Après action de l'amyloglucosidase pour éliminer le glycogène nous avons pu étudier l'évolution de la teneur en oses neutres conjugués. Les résultats obtenus sont groupés dans le tableau 2 .

\section{TABLEAU I}

Évolution de la teneur en glucides et en sulfates des ovocytes isolés de $\mathrm{P}$. cultrifera

Les valeurs sont exprimées en fonction du poids sec ovocytaire après précipitation alcoolique et lyophilisation

\begin{tabular}{|c|c|c|c|c|}
\hline \multirow{2}{*}{ Diamètre ovocytaire } & $130 \mu$ & $130-170 \mu$ & $180-220 \mu$ & $250 \mu$ \\
\hline & $\begin{array}{l}\text { Vitellus } \\
\text { protéique }\end{array}$ & \multicolumn{2}{|c|}{ Synthèses glucidiques } & $\begin{array}{l}\text { Maturité } \\
\text { épitoquie }\end{array}$ \\
\hline Glucides totaux $(\%) \ldots$ & 15,8 & 28,5 & 28,3 & 34,6 \\
\hline Oses neutres $(\%) \ldots \ldots$ & 10,1 & 21,6 & 20,6 & 27,4 \\
\hline Acides uroniques (\%) & 2,5 & 3,0 & 4,8 & 5,6 \\
\hline Acides sialiques . . . . & 0 & 0 & 0 & $\mathbf{0}$ \\
\hline Osamines $(\%)$ & 3,2 & 3,9 & 2,9 & 1,6 \\
\hline Sulfates $(\%) \ldots$ & 1,9 & 3,5 & 4,6 & 7,5 \\
\hline
\end{tabular}

TABLEAU 2

Importance quantitative de glycogène et des autres oses neutres ovocytaires lors de la maturation de $\mathrm{P}$. cultrifera

Le pourcentage est relatif au poids sec d'ovocytes

\begin{tabular}{c|c|c|c|c}
\hline \hline Diamètre ovocytaire & $130 \mu$ & $130-170 \mu$ & $180-220 \mu$ & $\begin{array}{r}250 \mu \\
\text { maturité }\end{array}$ \\
\hline & & & \\
Oses neutres totaux (\%).. & 10,1 & 21,6 & 20,6 & 27,4 \\
Glycogène ou polymères & 1,6 & 10,7 & 3,9 & 3,2 \\
de glucose (\%) ..... & $1,6,5$ & 10,9 & 16,7 & 24,2 \\
Oses neutr. conjug. (\%).. & 8,5 & & \\
\hline
\end{tabular}

Débarrassés du glycogène, les autres oses neutres conjugués ovocytaires présentent une évolution croissante et régulière comparable à celle des acides uroniques et des sulfates (tabl. 3). Les rapports entre ces différentes substances sont presque constants d'un stade à l'autre, seule l'importance quantitative de ce polysaccharide varie pendant la maturation cellulaire.

L'ovocyte de $P$. cultrifera synthétise donc un polysaccharide renfermant des oses neutres, des acides uroniques et des sulfates. La teneur ovocytaire de ce polysaccharide est en rapport direct avec l'activité inhibitrice du cerveau (fig. 7). La mesure quantitative de ce glucide donne une indication précieuse du taux d'inhibition cérébral et peut être considérée comme un test biochimique, qui complète le test biologique décrit précédemment, de l'activité endocrine des Néréidiens. 
TABLEAU 3

Rapports comparatifs entre les teneurs en oses neutres conjugués, en acides vironiques et sulfates des ovocytes de $\mathrm{P}$. cultrifera

\begin{tabular}{c|c|c|c|c}
\hline \hline Diamètre ovocytaire & $130 \mu$ & $130-170 \mu$ & $180-220 \mu$ & $\begin{array}{c}250 \mu \\
\text { maturité }\end{array}$ \\
\hline Rapport : $\frac{\text { acides uroniques }}{\text { oses neutres conjugués }}$ & 0,29 & 0,29 & 0,28 & 0,25 \\
\hline Rapport $: \frac{\text { sulfates }}{\text { oses neutres conjugués }}$ & 0,25 & 0,29 & 0,27 & 0,31 \\
\hline
\end{tabular}

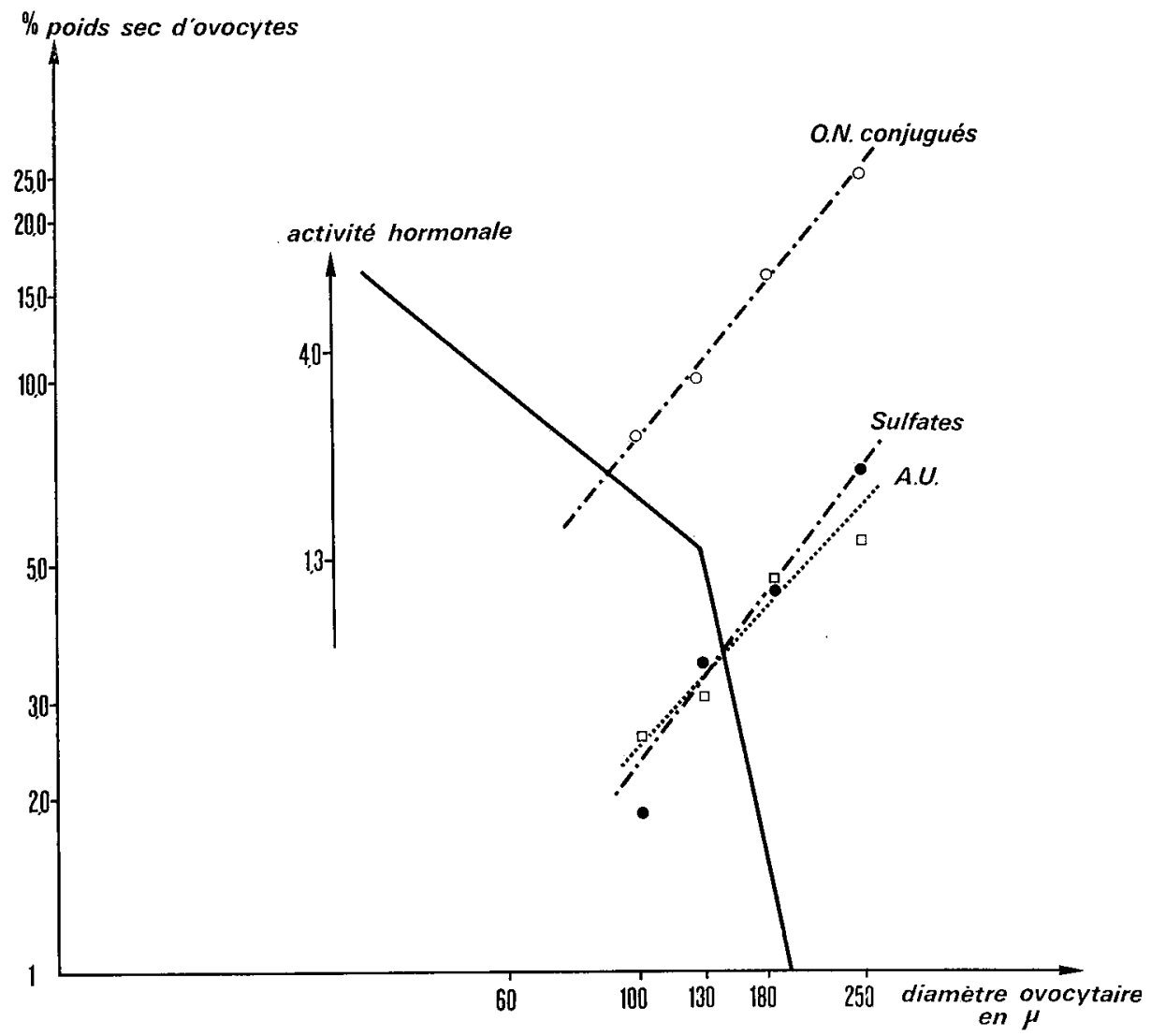

FIG. 7. - Rapports entre les niveaux d'activite hormonale et les teneurs en oses noutres conjugués (ON), en acides uroniques (AU) et en sulfates des ovocytes isolés. C'est la baisse de l'inhibition cérébrale qui conditionne l'importance des synthèses de ce polysaccharide sulfaté. 


\title{
CONCLUSION
}

Il est possible de distinguer deux étapes dans la maturation des ovocytes des Néréidiens, étapes soumises à un conditionnement hormonal précis et caractérisées par les synthèses spécifiques de réserves différentes. Dans chacune de ces étapes, l'appareil de Golgi et le nucléole jouant un rôle capital.

I. Dans un premier stade, l'ovocyte, influencé par une forte sécrétion endocrine cérébrale, élabore des lipides et le vitellus protéique. Ce dernier apparaît dans des dictyosomes de petite taille. Pauvre en glycogène, le jeune ovocyte ne renferme que peu d'autres polysaccharides. Son nucléole est caractérisé par la prédominance du composant granulaire ; de nombreuses extrusions nucléolaires sont émises à ce stade en direction du cytoplasme.

2. Puis, contemporaines de la chute de la production hormonale, commencent, dans les dictyosomes de grande taille, les synthèses de polysaccharides riches en acides uroniques et en sulfates (SPIK et PORCHE'T, sous presse). Ces substances, typiques de 1'ovocyte mature seront rejetées lors de la fécondation pour former la coque de l'œuf. Le glycogène, abondant au début de ce stade se raréfie au fur et à mesure de l'élaboration de ces polysaccharides. Le nucléole a acquis une structure à prédominance fibrillaire.

Le passage entre ces deux phases constitue une période critique pour l'ovocyte. C'est alors que l'activité endocrine diminue, que le nucléole et l'appareil de Golgi des ovocytes changent d'aspect et de fonction et que la teneur en glucides est doublée, par rapport au stade précédent dans le liquide cœlomique et dans les gamètes. Si ce passage est réalisé brutalement (suppression trop précoce du facteur hormonal par ablation du cerveau de l'animal, par exemple) l'ovocyte subit une dégénérescence.

Cette description schématique de la maturation ovocytaire chez les Néréidiens ne donne qu'un aperçu du phénomène dont certains aspects n'ont pas été mentionnés dans ce travail. Par exemple, on sait qu'un équilibre constant est maintenu entre 1'activité endocrine du cerveau et l'état génital par l'intermédiaire de rétroactions de nature chimique (PORCHET et CARDon, r972).

Il en résulte, dans les conditions naturelles, une harmonie entre tous ces facteurs qui seule peut conduire l'ovocyte des Néréidiens à la maturité complète.

\section{SUMMARY}

\author{
EFFECT OF CEREBRAL, ENDOCRINE ACTIVITY ON NEREID \\ (ANNELIDES POLYCHETES) OOCYTE MATURATION. \\ CYTOLOGICAL AND BIOCHEMICAL ASPECTS
}

Nereid oocyte maturation is studied from a cytological, biochemical and endocrinological point of view. First, the lipidic reserves and the proteic vitellus appear. The nucleolus presents a granular aspect at that time. During the following stage, dictyosomes elaborate polysaccharidic substances rich in uronic acids and sulphate. A biochemical analysis of these 
oocytes shows that the amount of glucides is doubled, as compared to the preceeding stage. The nucleolus loses its granular structure and becomes fibrillo-granular.

The complete sequence of these syntheses is only accomplished under an exact hormonal conditioning. It requires a regular decrease of brain endocrine production, which terminates at submaturity. The cerebral hormone inhibits sexual maturation, but its presence, at well defined activity levels, is necessary to the good development of oocyte metabolism.

\section{RÉFÉRENCES BIBL,IOGRAPHIQUES}

Allen M. J., I967. Nucleic acid and protein synthesis in the developing oocytes of the budding form of the syllid Autolytus edwardsi. Biol. Bull., 138, 287-302.

Bertout M., I972. Relations entre l'évolution nucléolaire et l'activité endocrine du cerveau au cours de l'ovogenèse de Nereis diversicolor. I. Ovogenèse naturelle. Z. Zellforsch. (sous presse).

Bertout M., Dhainaut A., I971. Étude cytochimique et autoradiographique de l'ovogenèse de $N$. diversicolor, dans les conditions naturelles et en l'absence d'hormone cérébrale. Gen. comp. Endocrinol., 17, 371-387.

BoIlly-MARER Y., r962. Inhibition des transformations hétéronéréidiennes par le cerveau d'espèces sans épitoquie (Néréidiens). C. R. Acad. Sci., 254, $2830-2832$.

Caro L. G., Palade G. E., 1964. Protein synthesis storage and discharge in the pancreate exocrine cells. An autoradiographic study. J. Cell Biol., 20, 473-495.

Casanova R., r955. Influence du prostomium sur la régénération caudale chez Platynereis massiliensis. C. R. Acad. Sci., 24, 1814-1816.

Choquet M., I962. Effet inhibiteur de l'hormone cérébrale sur l'évolution des cellules sexuelles chez Nereis pelagica. C. R. Soc. Biol., 156, I I12-1114.

ClaRK R. B., I956. The neurosecretory system of the Polychaete Nephtys and its role in reproduction. Abstr. XXth Intern. Physiol. Congr., Bruxelles, I78.

Clark R. B., 1966. The integrative action of a worm's brain. Symp. Soc. exper. Biol., U. S. A., $\mathrm{n}^{0} 20$, 345-379.

Clark R. B., 1969. Endocrine influences in Annelids. Gen. comp. Endocrinol., suppl. 2, 572-581.

Clark R. B., Bonney D. G., 1960. Influence of the supracesophageal ganglion on posterior regeneration in Neveis diversicolor. J. Embryol. Exp. Morphol., 8, II2-I 8 .

Clark R. B., Clark M. E., Ruston R. J. G., 1962. The endocrinology of regeneration in some errant Polychaetes. Mem. Soc. Encor., 12, Neurosecretion, 275-284, Acad. Press., London.

Clark R. B., Ruston R. J. G., I963a. The influence of brain extirpation on oogenesis in the polychaete N. diversicolor. Gen. comp. Endocrinol., 3, 529-541.

Clark R. B., Ruston R. J. G., 1963 b. Time of release and action of a hormone influencing regeneration in the Polychaete N. diversicolor. Gen. comp. Endocrinol., 3, 542-553.

ClaRK R. B., Scully U., 1963. Hormonal control of growth in N. diversicolor. Gen. comp. Endocrinol., 4, 82-90.

Dhainaut A., I964a. Contribution à l'étude du métabolisme de l'ARN, par incorporation de ${ }^{8} \mathrm{H}$-uracile, au cours de l'ovogenèse chez $N$. diversicolor. Bull. Soc. Zool. Fr., 89, 408-4I3.

Dhainaut A., I964 b. Étude, en culture organotypique, de l'influence de l'hormone cérébrale des Néréidiens sur les synthèses de DNA au cours de la spermatogenèse. C. R. Acad. Sci., 259, 46r-464.

Dhainaut A., I 966 . Étude ultrastructurale de l'evolution cytoplasmique au cours des premiers stades de l'ovogenèse chez N. pelagica. C. R. Acad. Sci., 262, 2616-2619.

Dhainaut A., r967. Étude de la vitellogenèse chez Nereis diversicolor par autoradiographie a haute résolution. C. R. Acad. Sci., 265, 434-436.

Dhainaut A., I 968 . Étude par autoradiographie à haute résolution de l'élaboration des mucopolysaccharides acides au cours de l'ovogenese de Nereis pelagica. J. Microscopie, 7, 1075-1080.

Dhainaut A., rg7oa. Étude cytochimique et ultrastructurale de l'évolution ovocytaire de Nereis pelagica.

I. Ovogenèse naturelle. Z. Zellforsch. mikr. Anat. Dtsch., 104, 375-389.

II. Ovogenèse expérimentale en l'absence d'hormone. Z. Zellforsch. mikr. A nat. Dtsch., 104, $390-404$.

Dhainaut A., I97o b. Étude en microscopie électronique et par autoradiographie à haute résolutioo des extrusions nucléaires au cours de l'ovogenese de Nereis pelagica. J. Microscopie, 9, 99-I 8.

Dhainaut A., 1972. Évolution nucléolaire au cours de l'ovogenèse de $N$. pelagica. I. Étude mnrphologique. J. Microscopie, 18, 67-84.

Dhainaut A., Porchet M., I967. Évolution ovocytaire en l'absence d'hormone cérébrale chez P. cultrifera. C. R. Acad. Sci., 284, 2807-2810.

Durchon M., 1948. Épitoquie expérimentale chez deux Polychètes : P. cultrifera et N. irrorata. $C . R$. Acad. Sci., 227, 157-158. 
Durchon M., I95I. L'ablation du prostomium provoque chez les Néréidiens la maturation précoce des produits génitaux mâles. C. $R$. Acad. Sci., 232, 442-443.

Durchon M., 1952. Recherches expérimentales sur deux aspects de la reproduction chez les Annélides Polychètes: l'épitoquie et la stolonisation. Ann. Sc. nat. Zool. Biol. anim., 14, Irg-2o6.

Durchon M., r956a. Rôle du cerveau dans la maturation génitale et le déclenchement de l'épitoquie chez les Néréidiens. Ann. Sc. nat. Zool. Biol. anim., 18, 269-273.

Durchon M., I956 b. Influence du cerveau sur les processus de régénération caudale chez les Néréidiens. Arch. Zool. Exp. gén., 94, 1-9.

Durchon M., r96o. L'endocrinologie chez les Annélides Polychètes. Bull. Soc. Zool. Fr., 85, $275-301$.

Durchon M., r967 a. L'endocrinologie des Vers et des Mollusques. Masson, Paris, 237 p.

Durchon M., I967 $b$. Rôle du système nerveux dans la régénération chez les Annélides. Bull. Soc. Zool. Fr., 92, 319-331.

Durchon M., Choquet M., I96r. Inhibition de l'épitoquie naturelle chez P. cultrifera. C. R. Acad. Sci., 155, 504-506.

Durchon M., Porchet M., r97o. Dosage de l'activité endocrine cérébrale au cours du cycle génital femelle chez Nereis diversicolor. C. R. Acad. Sci., 270, r689-169r.

Durchon M., Porchet M., I97I. Premières données quantitatives sur l'activité endocrine du cerveau des Néréidiens au cours de leur cycle sexuel. Gen. comp. Endocrinol., 16, 555-565.

Durchon M., Vivier E., I964. Influence des sécrétions endocrines sur le cycle des Grégarines chez les Néréidiens. Ann. Endoc., Paris, 25, 43-48.

Golding D. W., r967. Endocrinology, Regeneration and Maturation in Nereis. Biol. Bull., 138, 567577.

Gould M. C., Shroeder P. C., I969. Studies on oogenesis in the polychaete Annelid Nereis grubei. I. Some aspects of RNA synthesis. Biol. Bull., 136, 216-225.

Hauenschild C., r955. Photoperiodizität als Ursache des von der Mondphase abhängigen Metamorphose. Rhythmus bei dem Polychaeten Pl. dumerilii. $\quad Z$. Naturforsch., $10 \mathrm{~b}, 658-666$.

Hauenschild C., 1956. Hormonale Hemmung der Gerschlechtsreife und Metamorphose bei dem Polychaeten $P l$. dumerilii. Z. Naturforsch., 11 b, I25-132.

HaUenschild C., rg60a. Abhängigkeit der Regenerationsleitung von der inneren Sekretion im Prostomium bei $P l$. dumerilii. $Z$. Naturforsch., $15 \mathrm{~b}, 52-55$.

Hauenschild C., 1960 b. Lunar periodicity. Cold. Spring Symposia, Quant. Biol., 25, $491-497$.

Hauenschild C., r963. Postembryonale Entwicklungsteuerung durch ein Gehirn-Hormon bei $P l$. dumerilii. Verhandl. deutsch. Zool. Gesellsch. (München), 3-I 20.

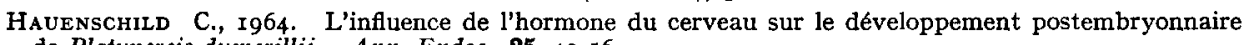
de Platynereis dumerillii. Ann. Endoc., 25, 49-56.

Hauenschild C., I965. Hormone bei Nereiden und anderen viederen Wirbellosen. Zoll. Jb., Abt. allg. Zool. Physiol. Tiere, Dtsch., 71, 5 I I-544.

Hauenschild C., 1966. Der hormonale Einfluss des Gehirns auf die sexuelle Entwicklung bei dem Polychaeta Platynereis dumerilii. Gen. comp. Endocrinol., 6, 26-73.

Hauenschild C., 197o. Beziekungen zwischen Gehir-hormon und Geschlechtsdifferenzierung bei dem proterandrischen Polychaeten Platynereis massiliensis. Zool. Anz., Suppl., Dtsch., n ${ }^{\circ}$ 33, $249^{-253 .}$

Herpin R., 1925. Recherches biologiques sur la reproduction et le développement de quelques Annélides Polychètes. Bull. Soc. Nat. Ouest Fr., $5,4^{\mathbf{e}}$ série.

JAmieson J. D., PALADE G. E., 1967. Intracellular transport of secretory proteins in the pancreatic exocrine cell. II. Transport to condensing vacuoles and zymogene granules. J. Cell Biol., 34, 597-615.

Malecha J., 1967. Transformation hétéronéréidienne et gamétogenèse chez Nereis succinea en culture organotypique. C. R. Acad. Sci., 265, 613-615.

Neutra M., Leblond C. P., I966. Synthesis of the carbohydrate of mucus in the golgi complex as shown by electron microscope radioautography of goblet cells from rat injected with glucose- $\mathrm{H}^{3}$. J.Cell. Biol., 30, II9-I36.

Porchet M., 1967. Rôle des ovocytes submatures dans l'arrêt de l'inhibition cérébrale chez $P$. cultrifera. C. R. Acad. Sci., 265, 1394-1 396.

Porchet M., I969. Interactions entre l'activité endocrine du cerveau et l'état génital chez $\mathrm{P}$. cultrifera. Thèse, 3 e Cycle, Lille, roo $\mathrm{p}$.

Porchex M., I970. Relations entre le cycle hormonal cérébral et l'évolution ovocytaire chez Perinereis cultrifera. Gen. comp. Endocrinol., 15, 220-231.

Porchet M., I972. Variation de l'activité endocrine des cerveaux en fonction de l'espèce, du sexe et du cycle vital chez quelques Néréidiens. Gen. comp. Endocrinol., 18, 276-283.

Porchet-Hennere E., ig69. Corrélations entre le cycle biologique d'une Coccidie Colotropha durchoni et celui de son hôte : Nereis diversicolor. $Z$. Parasitenk., 31, 299-314.

Porchet M., Cardon C., I972. Régulation de l'activité endocrine cérébrale chez les Neveidae (Annélides Polychetes). C. R. Acad. Sci., 275, 2375-2378.

Porchet M., Dhainaut A., 1969. Formation et migration des mucopolysaccharides au cours de l'ovogenèse de $P$. cultrifera et $N$. pelagica. Etude histochimique et ultrastructurale. $C$. $R$. Soc. Biol., 163, 4I8-420. 
Porchet M., Durchon M., I968. Influence de la maturité génitale sur la régénération postérieure chez $P$. cultrifera. C. R. Acad. Sci., 267, r94-ז96.

Schroeder P. C., 1971. Studies on oogenesis in the polychaete annelid Nereis grubei K. II. Oocyte growth rates in intact and hormone deficient animals. Gen. comp. Endocrinol., 16, 312-322.

SPIK G., Porchex M. Évolution quantitative et qualitative des glucides dans les ovocytes et le liquide cœlomique de $P$. cultrifera. Comp. Biochem. Physiol. (sous presse).

TwEedell K., I966. Oocyte development and incorporation of ${ }^{3} \mathrm{H}$-thymidine and ${ }^{3} \mathrm{H}$-uridine in $P$ ectinaria gouldii. Biol. Bull., 131, 516-538. 


\title{
PLANCHE I
}

\author{
FIG. 4
}

Élaboration du vitellus protéique $(N$. pelagica). Les éléments de la face proximale (FP) de l'appareil de Golgi (G) présentent un aspect dilaté. A proximité de la face distale (FD) on constate la présence d'un globule de vitellus (V) en formation.

(mv et ch : microvillosités et chorion de 1'enveloppe ovocytaire)

$$
\times 32000
$$

FIG. 5

Incorporation de glucose- ${ }^{-3} \mathrm{H}$ pendant 6 heures dans des ovocytes de $N$. pelagica submatures (I70 $\mu$ de diamètre).

La radioactivité est localisée au niveau des saccules golgiens (G) ; EO : enveloppe ovocytaire; $\mathrm{MC}$ : polysaccharides. 
4

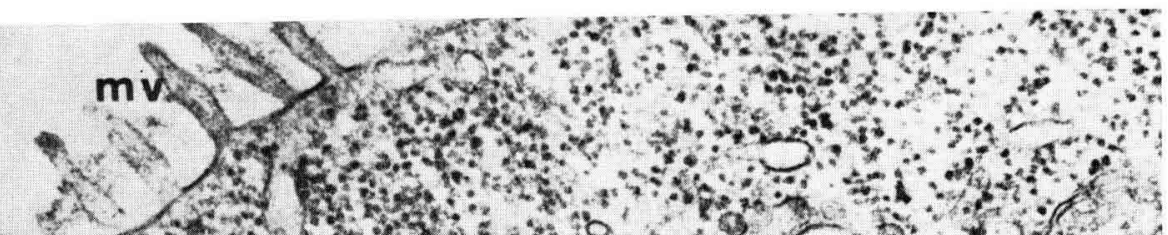

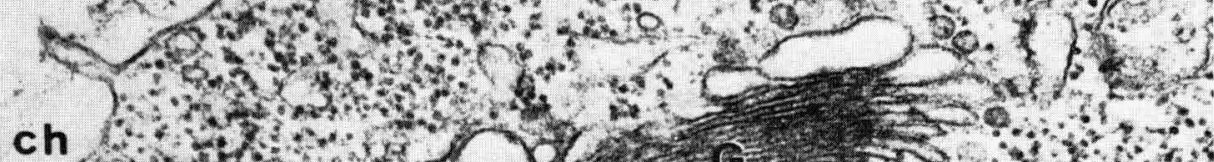

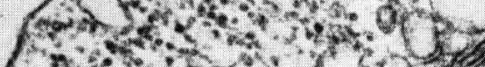

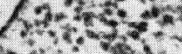

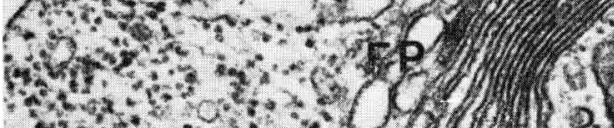

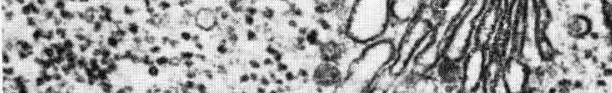

1.

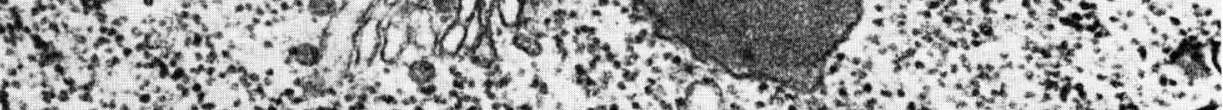

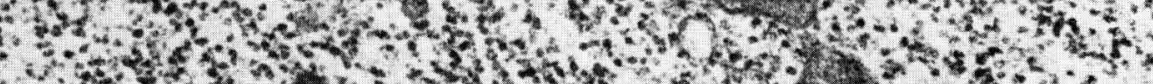

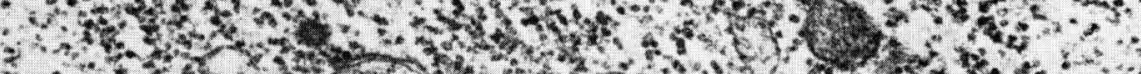

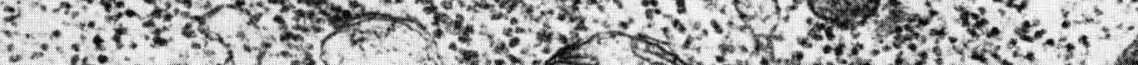

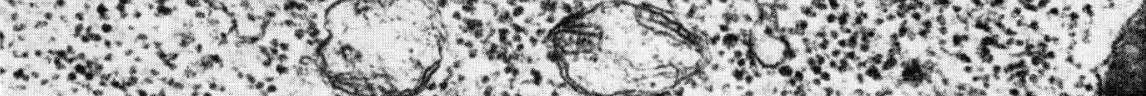

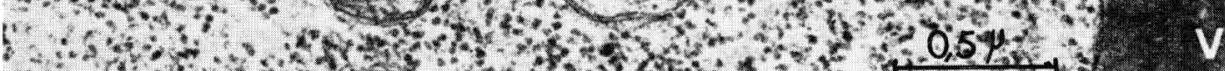

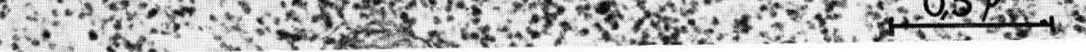

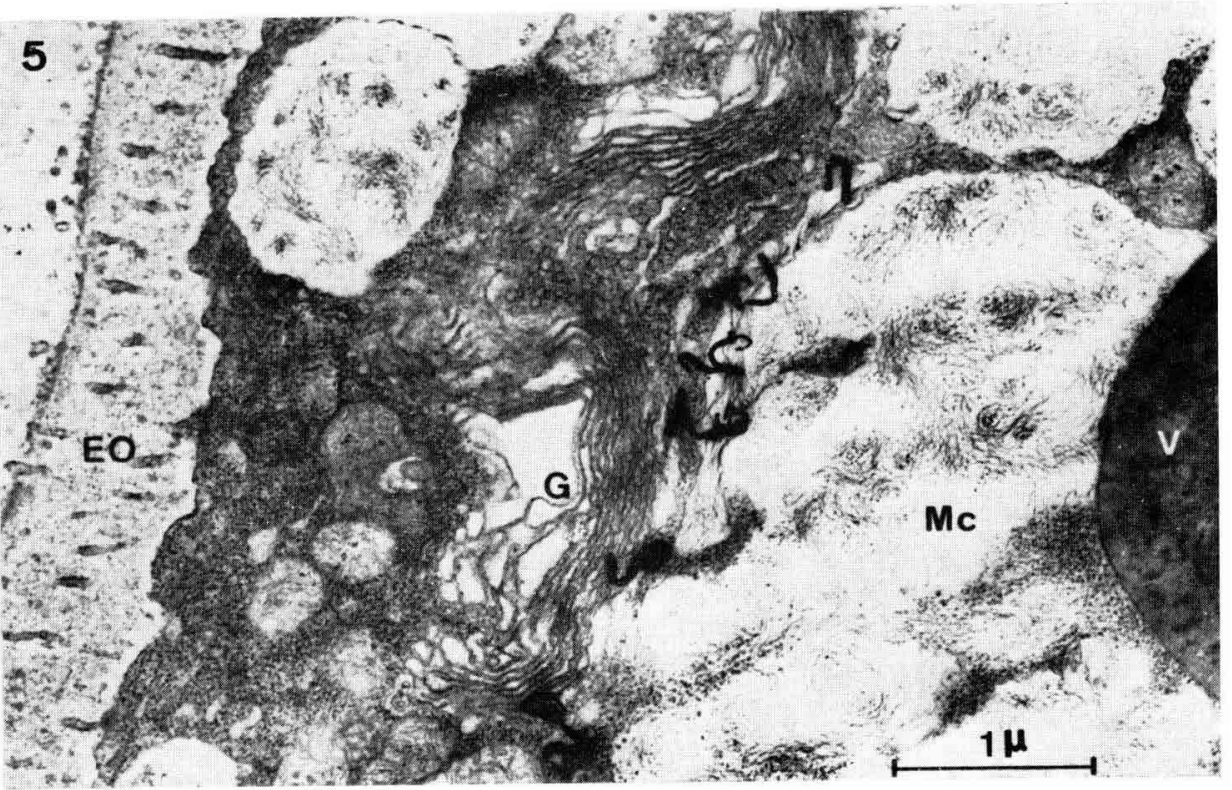

